\title{
The stabilizing role of itinerant ferromagnetism in inter-granular cohesion in iron
}

\author{
D. Yeşilleten $\dagger$, M. Nastar $\ddagger$, T. A. Arias $\dagger$, A.T. Paxton $\ddagger$, S. Yip $\ddagger$ \\ Departments of Physics $\nmid$ and Nuclear Engineering $\ddagger$ \\ Massachusetts Institute of Technology Cambridge, MA 02139
}

\begin{abstract}
We present a simple, general energy functional for ferromagnetic materials based upon a local spin density extension to the Stoner theory of itinerant ferromagnetism. The functional reproduces well available $a b$ initio results and experimental interfacial energies for grain boundaries in iron. The model shows that intergranular cohesion along symmetric tilt boundaries in iron is dependent upon strong magnetic structure at the interface, illuminates the mechanisms underlying this structure, and provides a simple explanation for relaxation of the atomic structure at these boundaries.

Iron and its steel alloys, exhibit two distinct outstanding physical properties, high strength and high magnetic response. Little is known about the direct relationship, if any, between the microscopic origins of these properties. The exchange interaction, preference for alignment of spins to reduce interelectronic Coulomb repulsion through the statistical avoidance of Fermions, drives itinerant ferromagnetism. Fundamental topological excitations of the crystalline lattice govern mechanical response: dislocations mediate plasticity, grain boundaries control microstructure and inter-granular cohesion. In this letter we present a new model for itinerant ferromagnetic iron and demonstrate that the exchange interaction plays a significant stabilizing role in grain boundaries, thereby contributing to the strength of inter-granular cohesion.
\end{abstract}

The fundamental role played by exchange in controlling the mechanical response of magnetic materials is at present poorly understood for lack of an appropriate microscopic theory. Studies in iron to date are limited to either full-blown $a b$ initio spindependent electronic structure calculations [1] or simple interatomic potentials such as the embedded atom method (EAM) [2]. While describing all of the correct physics, $a b$ initio calculations treat explicitly too many degrees of freedom to allow studies of the complex structures of all but the simplest extended defects. For this reason, total energy ab initio studies of iron to date have been limited to small clusters encompassing the behavior of the simplest $(\Sigma=3(111))$

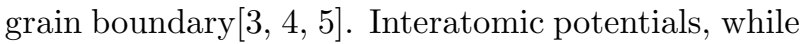
practical for complex systems, deal with too few degrees of freedom to treat itinerant exchange properly. Nonetheless, these potentials have been useful in studies of grain boundaries[6], dislocations [7] and even fracture 8]. Energies calculated for grain boundaries with the EAM[6], however, are exaggerated by about a factor of two when compared with experimental values [9], beginning to approach even the experimental surface energy 10. Here, we present a simple, general model which gives much better results and reveals the mechanisms stabilizing the boundaries.

In response to the above weakness, Krasko 11 has introduced recently an atomistic potential which includes a prescription for estimating the exchange energy of each atom from its local environment. Here, we follow an alternate, microscopic route. In accord with the microscopic origin of itinerant ferromagnetism, we consider the extended nature of the electrons and determine only the spin-moment coupling constant, the Stoner parameter 12], as a function of the local atomic environment.

Microscopic Approach - Such intermediate, electronic structure based descriptions of iron have been developed in previous studies of iron 13, 14, but for ideal crystalline systems. Our approach to the study of defects is to first identify the smallest, physically reasonable set of degrees of freedom from these studies, and then extend this treatment to include inhomogeneous systems.

Hasegawa and Pettifor 13 reproduce the experimental $P-T$ phase diagram of iron by combining a tight-binding description of the $d$-bands with a treatment of spin-fluctuation effects. They note that as $T \rightarrow 0$, spin fluctuation effects become unim- 
portant and their spin-fluctuation theory reduces to the traditional mean-field Stoner theory of itinerant ferromagnetism 12]. They identify the temperature at which this happens to be $T_{f} \approx 500 \mathrm{~K}$. Zhong, Overney, and Tomanek 14 take up the fact that mean-field Stoner theory is sufficient at room temperature, and build a model for bulk crystalline iron based upon an spd tight-binding Hamiltonian and Stoner theory, treating only the mean atomic spins.

We therefore identify a minimal set of active physical degrees of freedom below $T_{f}$ to consist of the net mean spin moment on each atom and single particle states constructed from atomic-like $d$-orbitals. To pass beyond perfect crystalline material, we introduce a local atomic spin-density extension to Stoner theory.

Construction of Energy Functional - Our selected degrees of freedom are (1) the linear combination coefficients $\psi_{n k}(i, m)$ describing the bonding among the $m_{z}=-2, \ldots, 2$ atomic $d$-states of each atom $i$ for each band $n$ and each point in the Brillioun zone $k$, (2) the net spin $n_{\sigma}(i), \sigma= \pm 1$, associated with each atom $i$, and (3) the location $\vec{\tau}_{i}$ of each atom $i$.

To describe the bonding contribution to the energy $\epsilon_{n k}$ associated with each single-particle orbital, we use an orthogonal two-center tight-binding Hamiltonian description 15. In the usual fashion, we take the diagonal elements of the matrix of hopping integrals among $L_{z}$ eigenstates for two atoms separated by a distance $\tau$ along the z-axis, $d d \delta, d d \pi, d d \sigma, d d \pi, d d \delta$, to decay exponentially with distance, $d d \lambda=d d \lambda_{o} e^{-q \tau}$. To set the primitive matrix elements $d d \lambda_{o}$ and the decay factor $q$ we insist that the tight-binding Hamiltonian reproduce well the $a b$ initio density of states for the $d$-bands, which is accomplished by setting the Slater-Koster parameters to $d d \sigma_{o}: d d \pi_{o}: d d \delta_{o}=-$ 1 Ryd:1 Ryd:-0.5 Ryd and the decay factor to $q=$ 0.63872 .

In summing over the single particle energies, we replace the integral over the Brillioun zone with a discrete sum with appropriate weights $w_{k}[16]$. The tendency of our system to spin-polarize requires us to consider separate Fermi occupation numbers $f_{n k \sigma}$ for each spin channel $\sigma$. We also include the Fermionic entropy

$s_{n k \sigma} \equiv-k_{B}\left[f_{n k \sigma} \ln f_{n k \sigma}+\left(1-f_{n k \sigma}\right) \ln \left(1-f_{n k \sigma}\right)\right]$

so that our band structure energy appears as the first sum in the energy functional (1) where the $\epsilon_{n k}$ are the eigenvalues of the tight-binding Hamiltonian. All of the results below are computed at $T=300^{\circ} \mathrm{K}$.

Combining the Fermi occupations $f_{n k \sigma}$ with the expansion coefficients $\psi_{n k \sigma}$ yields the local atomic spin densities, $n_{\sigma}(i) \equiv \sum_{n, k, m} w_{k} f_{n k \sigma}\left|\psi_{n k}(i, m)\right|^{2}$. The Stoner theory of itinerant ferromagnetism describes an energetic benefit of polarization due to exchange of magnitude $N_{a t} I m^{2} / 4$, where $N_{a t}$ is the total number of atoms in the crystal, $m$ is the spin polarization per atom and $I$ is the Stoner parameter 12 . To extend this theory beyond homogeneous bulk systems, we introduce a local approximation to the exchange energy in the same spirit as the local-density approximation 17] of density functional theory 18. In particular, for an inhomogeneous system, we associate a separate energy contribution to the exchange from each atom equal to what we would expect on a per atom basis from a homogeneous system consisting of atoms in the identical environment with identical spin polarization. This contribution appears as the second sum in our energy functional (11). The physical motivation for this approximation is that the itinerant nature of magnetism in iron tends to smooth variations in the spin polarization, limiting the effects of gradient corrections. This approach has the advantage of allowing us to draw upon $a b$ initio values of the Stoner exchange parameter in bulk.

Krasko 19 has performed ab initio linear response theory calculations of the Stoner parameter $I$ in bcc and fcc bulk iron and found it to have a mild, approximately linear volume dependence, $d I / d s=-0.01 \mathrm{Ry}-$ dberg/Bohr where $s$ is the Wigner-Seitz (WS) radius, and to have slightly different values for the bcc and fcc lattices, $I_{b c c}^{o}=0.072 \mathrm{Ryd} / \mu_{B}^{2}$ and $I_{f c c}^{o}=$ $0.069 \mathrm{Ryd} / \mu_{B}^{2}$ at $s=2.66 \mathrm{Bohr}$. The use of these values in our model gives the correct magnetic and non-magnetic states for the bcc and fcc structures, respectively. However, to yield the correct total energy ordering of states, we have found necessary a slight enhancement of the bulk Stoner parameters to $I_{b c c}^{o}=0.077 \mathrm{Ryd} / \mu_{B}^{2}$ and $I_{f c c}^{o}=0.070 \mathrm{Ryd} / \mu_{B}^{2}$, which leads to no magnetization in the fcc phase and a bcc phase magnetic moment of $2.56 \mu_{B}$ /atom, somewhat enhanced relative to the accepted moment of $2.2 \mu_{B}$ /atom.

Going beyond bulk to inhomogeneous systems with intermediate coordinations $Q$, we make a linear interpolation for $I_{i}$ between what would be expected at the same WS radius for the $Q_{b c c}=8$ and the $Q_{f c c}=12$ lattices. We determine the local coordination number $Q_{i}$ and WS radius $s_{i}$ for each atom $i$ with the formulation developed by Sawada 20 and the conversion $s_{i}=R_{i}\left(a+b / Q_{i}+c / Q_{i}^{2}\right) / 2$ from his parameter $R_{i}$. We find that setting Sawada's coefficients to $\lambda_{1} \equiv 4.5023 \mathrm{Bohr}^{-1}, \lambda_{2} \equiv 10.6376 \mathrm{Bohr}^{-2}$ and using $a \equiv 1.7144, b \equiv-9.0948, c \equiv 56.372$ reproduces to within $0.01 \%$ the correct coordination 
numbers and to within $0.3 \%$ the correct WS radii for the diamond structure, bcc and fcc lattices packed at the atomic density of bcc iron. With the Stoner parameters thus determined, we set the filling $N_{d}$ of the manifold of $d$-states, so that the Fermi level for spindown electrons in the bcc structure falls precisely at the minimum of the pseudo-gap in the tight-binding density of states, reproducing the physical behavior observed in $a b$ initio calculations. The resulting filling, $N_{d}=6.7$ electrons/atom, is in good agreement with the value of $N_{d}=7.0$ used successfully in [13].

Finally, for the energy associated with the locations of the atoms, we take a power-law relationship between the interatomic potential and the hopping elements, a standard successful practice in tight-binding calculations 21], to produce the final term in (11). We fit the two parameters $b$ and $p$ to the experimental equilibrium lattice constant and bulk modulus for the bcc phase of iron, yielding $p=2.2355$ Bohr $^{-1}$ and $b=872.5174$ Ryd, respectively. The ratio $\lambda \equiv p / q$ corresponds to a normalized hardness 21, $\alpha_{h} \equiv(\lambda-1) / \lambda \approx 0.7$, in line with the values near two-thirds observed previously in tight-binding descriptions of the transition metals [22].

Our final energy functional is thus,

$$
\begin{aligned}
E\left(\left\{\vec{\tau}_{i}\right\}\right)= & \min _{\psi, f}^{\prime}\left\{\sum_{n k \sigma} w_{k}\left(f_{n k \sigma} \epsilon_{n k}-T s_{n k \sigma}\right)\right. \\
& -\frac{1}{4} \sum_{i} I_{i}\left(n_{\uparrow}(i)-n_{\downarrow}(i)\right)^{2} \\
& \left.+\frac{b}{2} \sum_{i \neq j} e^{-p \tau_{i j}}\right\}
\end{aligned}
$$

The constraints on the minimization are Fermi statistics, $0 \leq f_{n k \sigma} \leq 1$ and the total number of $d$-electrons, $N_{d}=\sum_{n k \sigma} w_{k} f_{n k \sigma}$. This formulation is equivalent in bulk systems to the familiar formulation of the Stoner theory in terms of a rigid shift between the up and down electronic density of states. The present formulation, however, has the advantage in treating complex structures of allowing distinct local Stoner parameters to be applied to each atom according to its environment. Finally, stationarity of the energy functional with respect to the fillings dramatically simplifies the evaluation of forces.

Verification - To confirm the applicability of our description to iron, we discuss briefly the comparison of our results with available $a b$ initio and experimental information for the bulk crystalline phases and for grain boundaries in bcc iron.

In bulk, we reproduce the correct sequence bcchcp-fcc of phases finding $E_{h c p}-E_{b c c}=2.2 \mathrm{mRyd} /$ atom and $E_{f c c}-E_{b c c}=6.5 \mathrm{mRyd} /$ atom. Our fcc-bcc energy difference, is on the order of what is found in other calculations [13, and theoretical and experimental extrapolation 23, 24. In agreement with $a b$ initio calculations 25, 26, we observe that the hcp phase is more stable than the non-magnetic fcc phase for all values of the WS radius. We predict a pressure-induced phase transition from the bcc to the hcp phase at a WS radius of $s=2.6$ Bohr, in good agreement with the $a b$ initio studies of 24, 27. (Note that we did not fit our parameters to produce the previous two properties.) The small energy differences among these phases opens the question of mechanical stability. Our Hamiltonian gives a mechanically stable ferromagnetic bcc phase, even along the Bain transformation $\left(C^{\prime}>0\right)$. Our predicted $C^{\prime}$ and $C_{44}$, which we have made no attempt to fit, are about $25 \%$ lower than observed experimentally, corresponding to an underestimation of about $12 \%$ in the frequencies in the long-wavelength portion of the phonon spectrum.

The literature presents an experimental determination of an average typical grain boundary energy in $\alpha$-iron and $a b$ initio results for the spin moment distribution of the $\Sigma=5(310)$ and $\Sigma=3(111)$ boundaries and the atomic relaxation of the $\Sigma=3(111)$ boundary in iron. Table 11 summarizes our results for these and two other symmetric tilt boundaries. Our calculations were carried out in supercells containing two oppositely oriented boundaries separated by at least eighteen layers of atoms. We performed full structural and supercell relaxations of these boundaries.

Table 1 shows that our energy results are in good agreement with the experimental studies, which set the mean typical grain boundary energy to be approximately $770 \mathrm{erg} / \mathrm{cm}^{2}[9$. The $\Sigma=3(112)$ boundary, the coherent twin, is a known special case which is expected to be unrepresentatively low in energy. The magnetic and structural predictions of our energy functional are in excellent quantitative agreement with the $a b$ initio predictions for the outward structural relaxation of the atomic planes of the $\Sigma=3(111)$ boundary and for the fractional enhancement of the spin moments of the symmetry plane for both the $\Sigma=5(310)$ and $\Sigma=3(111)$ boundaries. (See $\Delta z$ and $\Delta m$ in Table 1).

Finally, figure 11 presents a more detailed comparison with $a b$ initio calculations, showing the spatial distribution of spin moments in the vicinity of the $\Sigma=5(310)$ boundary. The figure shows that our model not only reproduces the enhancement of moments on the symmetry plane but also predicts correctly the tendency for the spin to fall below the bulk 

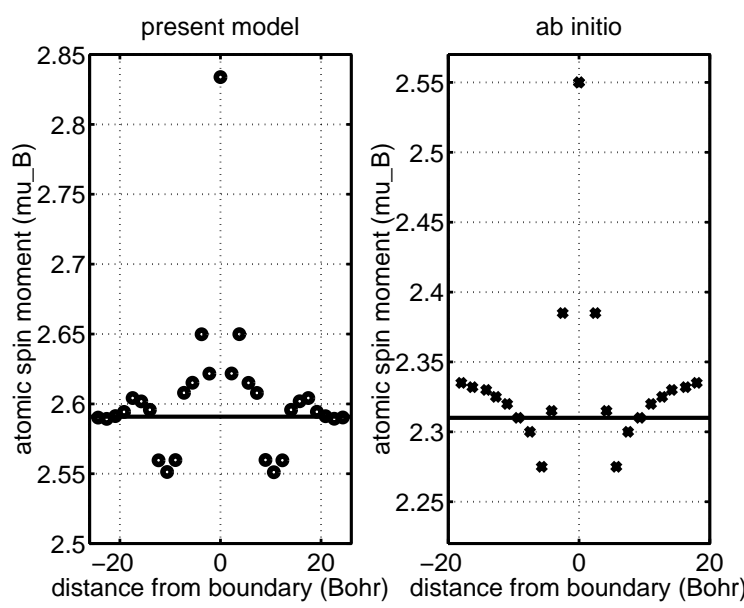

Figure 1: Prediction of atomic spin moments in the vicinity of the $\Sigma=5(310)$ symmetric tilt boundary in iron. Left: present model, Right: ab initio results. Horizontal lines give the bulk phase moment of the respective calculation.

moment before eventually healing back to the bulk value as one moves away from the boundary. Some discrepancies become apparent in the results at this high level level of detail. This comparison serves to underscores the fact that there are limitations to any simplified, semiempirical model. Nonetheless, the overall level of agreement which we have found supports the fundamental soundness of our approach and its ability to give accurate predictions of global quantities and physical trends for complex structures in iron.

Exchange stabilization of grain boundaries in iron - The fact that the embedded atom model consistently exaggerates boundary energies in iron by a factor of two over our functional points to the participation in boundary energetics of a microscopic mechanism more subtle than simple distortions of the metallic bonding network. The data in Table 1 show that the exchange interaction is a major factor in the physics of the grain boundaries. The tendency to minimize the exchange contribution drives the system to lower its energy at the expense of an almost compensating dramatic increase in the atomic and band structure contributions. The end result of this balance is the lower and much more realistic set of boundary energies in Table fi.

Breakdown of the large negative contributions from the exchange interaction shows that the enhancement comes mostly from an increase by $10-20 \%$ of the atomic spin moments with a lesser contribution from an increase by $2-4 \%$ of the Stoner exchange parameters themselves. (Table 1.) The increase in magnetic moments along the boundary therefore plays the key role in the large stabilizing exchange interaction.

The origin of this increase is the fact that the states which contribute to the spin moments in the system tend to be more localized on the grain boundary. States with energy below both Fermi levels $\mu_{\uparrow}$ and $\mu_{\downarrow}$ are filled equally with up and down spins and so contribute nothing to the net moment, while those states with energy above both Fermi levels are completely empty. Only those states between these two Fermi levels are filled with uncompensated spins and contribute to the spin moments. The range of energies between the two Fermi levels is near the center of the $d$-band. The states in this energy range tend to localize on the boundary as a direct consequence of the more open structure of the boundary, which lowers the tight-binding matrix elements $d d \lambda$ and thereby narrows the band toward the band-center. We confirmed this latter behavior by direct inspection of the electronic states.

Finally, our model also sheds light on the geometric relaxation of the grain boundaries. In all boundaries in our study, the two planes of atoms immediately neighboring the symmetry plane to relax outward and compress into the surrounding bulk. Because the Stoner parameters increase with decreasing atomic volume $(d I / d s<0$, above) this diminishes the Stoner parameter on the symmetry plane but enhances the exchange parameter in a total of four planes, one pair on either side of the boundary. This relaxation pattern enhances the exchange stabilization by sacrificing the exchange parameters on the single symmetry plane in favor of a total of four nearby planes.

In conclusion, we have developed a local spin density functional description of itinerant ferromagnetic materials which provides a simple and accurate picture of the relationships among geometry, electronic structure and stability of tilt grain boundaries in iron.

\section{Acknowledgments}

This work was supported by the MRSEC Program of the National Science Foundation (DMR 94-00334) and by the Alfred P. Sloan Foundation (BR-3456). Computational support provided by the MIT Xolas prototype SUN cluster.

\section{References}

[1] M.C. Payne, et al., Rev. Mod. Phys. 64, 1045 (1992). 
[2] M.W. Finnis and J.E. Sinclair, Phil. Mag., A50, 45 (1984) and A53, 161 (1984).

[3] G.L. Krasko, Defect - Interface Interactions Symposium, Boston MA, Mater. Res. Soc; Pittsburgh, PA (1993), p. 369.

[4] Y. Itsumi, D.E. Ellis, J. Mat. Res. 11, 2214 (1996).

[5] Ruqian Wu, A.J. Freeman, G.B. Olson, Phys. Rev. B53, 7504 (1995).

[6] R. Watanabe, A. Nogami, T. Matsumiya, Materials Science Forum 204-206, 337 (1996).

[7] D. Farkas and P. L. Rodriguez, Scripta Metall. 30, 921 (1994).

[8] K. S. Cheung and S. Yip, Modell. Simul. Mater. Sci. Eng. 2, 865 (1994).

[9] L.H. Van Vlack, Trans. Amer. Inst. Min. Met. End. 191, 251 (1951).

[10] W. Missol, Act. Met. 24, 73 (1976).

[11] Genrich L. Krasko, J. Appl. Phys. 79, 4682 (1996).

[12] E. C. Stoner, Proc. R. Soc. London, Ser. A169, $339,(1939)$.

[13] H. Hasegawa and D.G. Pettifor, Phys. Rev. Lett. 50130 (1983).

[14] W. Zhong, G. Overney, D. Tomanek, Phys. Rev. B 47, 95 (1993).

[15] J.C. Slater and G.F. Koster, Phys. Rev. B39, 1498 (1954).

[16] H.J. Monkhorst and J.D. Pack, Phys. Rev. B13, 5188 (1976).

[17] W. Kohn and L.J. Sham, Phys. Rev. 140, 1133A (1965).

[18] P. Hohenberg and W. Kohn Phys. Rev. 136, 864B (1964).

[19] G. L. Krasko, Sol. Stat. Comm. 70, 1099 (1989).

[20] S. Sawada, Vacuum 41, 612 (1990).

[21] D. Pettifor, Bonding and Structure of Molecules and Solids, Claredon Press, Oxford (1995), p. 7879 .

[22] Ibid, p. 188.
[23] L. Kaufman and H. Bernstein, Computer Calculations of Phase Diagrams (Academic Press, New York, 1970)

[24] W. Bendick, W. Pepperhof, Acta Metall, 30, 679 (1982)

[25] A. T. Paxton, M. Methfessel, H. M. Polatoglou, Phys. Rev. B 41, 8127 (1990).

[26] L. Stixrude, R. E. Cohen, D. J. Singh, Phys. Rev. B 50, 6442 (1994).

[27] P. Soderlind, R. Ahuja, O. Eriksson, J. M. Wills, B. Johansson, Phys. Rev. B 50, 5918 (1994)

[28] K. Hampel, D.D. Vvedensky and S. Crampin, Phys. Rev. B47, 4810 (1993). 


\begin{tabular}{cccccc} 
Boundary & $\begin{array}{c}E_{g b} \\
\mathrm{erg} / \mathrm{cm}^{2}\end{array}$ & $\begin{array}{c}E_{x c} \\
\mathrm{erg} / \mathrm{cm}^{2}\end{array}$ & $\Delta m$ & $\Delta I_{\max }$ & $\begin{array}{c}\Delta z \\
\mathrm{Bohr}\end{array}$ \\
\hline$\Sigma=3(112)$ & $\begin{array}{c}140 \\
\left(300^{* *}\right)\end{array}$ & -8600 & $14 \%$ & $2 \%$ & 0.1 \\
\hline$\Sigma=5(310)$ & $\begin{array}{c}560 \\
\left(1300^{* *}\right) \\
\left(\sim 770^{*}\right)\end{array}$ & -3800 & $9 \%$ & $3 \%$ & 0.5 \\
\hline$\Sigma=3(111)$ & 770 & -5200 & $\left(8 \%^{\dagger}\right)$ & & \\
\hline$\Sigma=9(114)$ & $\begin{array}{c}76 \% \\
\left(1450^{* *}\right) \\
\\
\left(\sim 770^{*}\right)\end{array}$ & -4100 & $15 \%$ & $4 \%$ & 0.6 \\
& & & & $\left.0.5-0.8^{+}\right)$ \\
\hline \hline
\end{tabular}

Expt: ${ }^{*}\left[\right.$ [], EAM: ${ }^{* *}[6] ;$ Ab initio: ${ }^{\dagger}[28],{ }^{+}[3],{ }^{+}[5]$.

Table 1: Summary of Grain Boundary Results: Boundary formation energy $\left[E_{g b}\right]$, exchange contribution to the energy $\left[E_{e x}\right]$, change in magnetic moment on the boundary plane $[\Delta m]$, maximum Stoner parameter $\left[\Delta I_{\max }\right]$, outward motion of planes immediately neighboring the boundary $[\Delta z]$. Results of other studies appear in parenthesis. 